\title{
Fractal Approach to Alternating Current Impedance Spectroscopy Studies of Carbon Nanotubes/Epoxy Polymer Composites
}

\author{
Rajae Belhimria ${ }^{1}$, Sofia Boukheir ${ }^{1,2}$, Zineb Samir $^{1}$, Adel Len ${ }^{3,4}$, Mohammed Essaid Achour ${ }^{1, *}$, \\ Nandor Éber ${ }^{3}$, Luis Cadillon Costa ${ }^{5}$, Amane Oueriagli $^{2}$ \\ ${ }^{1}$ Laboratoire LASTID, Faculté des Sciences, Université Ibn Tofail, 14000 Kénitra , Morocco \\ ${ }^{2}$ Laboratoire LN2E, Faculté des Sciences, Université Cadi Ayyad, 40000 Marrakech, Morocco \\ ${ }^{3}$ Institute for Solid State Physics and Optics, Wigner Research Centre for Physics, \\ Hungarian Academy of Sciences, Budapest, P.O.Box 49, Hungary \\ ${ }^{4}$ Faculty of Engineering and Information Technology, University of Pécs, 7624 Pécs, Hungary \\ ${ }^{5}$ I3N and Physics Department, University of Aveiro, 3810-193 Aveiro, Portugal
}

*Correspondence to:

Achour ME,

Tel: +212-5-37-32-94-00

Fax: $+212-5-37-32-94-33$

E-mail:

achour.me@univ-ibntofail.ac.ma

Received August 1, 2017

Revised August 26, 2017

Accepted August 28, 2017
The dielectric relaxation characteristics of composites with different concentrations of carbon nanotubes loaded in an epoxy polymer matrix has been studied as a function of frequency over a wide range $(1 \mathrm{~Hz} \sim 10 \mathrm{MHz})$ at room temperature. Two characterization techniques were used in this work to measure and calculate the dimensionality parameters: small angle neutron scattering and impedance spectroscopy. The results obtained from both methods are in good agreement, indicating the reliability of the estimated fractal dimension, despite of the difference in the length scales accessed by the two techniques.

Key Words: Fractal dimension, Carbon nanotubes, Small angle neutron scattering, Impedance spectroscopy

\section{INTRODUCTION}

Since the documented discovery of carbon nanotubes (CNT), many researchers have sought to manufacture advanced composite CNT materials that exhibit one or more of their unique physical (mechanical, thermal, and electrical) properties (Ali \& Mousa, 2016; Han \& Fina, 2011; Sahoo et al., 2010). Polymer based composites have been considered the most suitable for further reinforcement with CNT (Cooper et al., 2002). Progress in reinforcing polymers with CNT has recently been reported for rubbery epoxy matrices (CNT/ epoxy) (Allaoui et al., 2002; Lau et al., 2002). Epoxy resins have excellent heat resistance, high strength, good stiffness, chemical resistance; therefore, they are applied in the field of coatings, casting, composites, laminates and encapsulation of semiconductor devices (El-Tantawy \& Deghaidy, 2000; Kang et al., 2001; Park et al., 2000).

The fractal geometry is a mathematical concept that describes objects of irregular shape. It has been used to analyze different objects in various scientific and technological branches since their description by Mandelbrot (Mandelbrot et al., 1984). Application of the fractal concept for the prediction of the physical properties of composites has demonstrated that the fractal dimension is related to the dielectric relaxation process (Hopkins et al., 2011; Lira-Olivares et al., 2000). The correlation of the insulator-conductor transition of composite materials with the fractal dimension of the aggregates has been studied in some works (Boukheir et al., 2016; Salome \& Carmona, 1991). Fractal dimension of a certain surface can, in principle, be inferred from different ways. These include, but are not limited to: surface image analysis, small angle neutron scattering (SANS) and impedance spectroscopy (IS). The latter two methods are especially widespread and used in different areas of research (Hattenhauer et al., 2015; Tagmouti et al., 2015; Tapasztó et al., 2014). The aggregated fillers in IS have constant phase element (CPE) behavior, i.e. they show

(a) This is an open-access article distributed under the terms of the Creative Commons Attribution Non-Commercial License (http://creativecommons.org/licenses/by-nc/4.0) which permits unrestricted noncommercial use, distribution, and reproduction in any medium, provided the original work is properly cited.

Copyrights @ 2017 by Korean Society of Microscopy 
capacitance dispersion. The value of the CPE exponent $n$ can be determined from the magnitudes of the impedance.

The present work, reports the results of a study whose main aim was to examine and compare performances of SANS and IS in determination of fractal properties and to establish the degree of correlation between the fractal dimensions obtained by these methods.

\section{MATERIALS AND METHODS}

\section{Materials}

Multiwalled carbon nanotubes (MWCNT) with the average diameter of $50 \mathrm{~nm}$ and the length in the range of 10 20 were adopted, in which the purity is higher than $95 \mathrm{wt} \%$. DGEBA (diglycidylic ether of bisphenol A) was used as the matrix. The neat polymer has a density of $1.19 \mathrm{gcm}^{-3}$, a DC conductivity of $1.4 \times 10^{-14} \mathrm{Sm}^{-1}$ and a glass transition temperature of about $60^{\circ} \mathrm{C}$. The contents of MWCNTs against DGEBA in the composites were from $0.2 \%$ to $5.0 \%$. MWCNT were mixed with the epoxy in different concentrations and stirred $\phi$ at room temperature. The MWCNT were first dispersed in methanol solution under magnetic agitation to reduce the maximum size of the aggregates. After complete evaporation of methanol, the obtained MWCNT powder was then directly added to the epoxy resin bisphenol A/aromatic hardener mixture. Finally, it was injected into sample moulds. After achieving gelation, the samples were unmolded after a few hours, then they were left in rest in order to reach the complete polymerization. Ten DGEBA/MWCNT composite samples were prepared and measurement results of six of them were chosen to be analyzed in this work.

\section{Electrical Impedance Measurements}

The temperature dependent alternating current (AC) impedance spectra were measured by using a Novocontrol Alpha-A Analyzer (Novocontrol Technologies, Hundsangen) combined with the impedance interface ZG4. Impedance measurements were carried out at $25^{\circ} \mathrm{C}$, in the frequency range from $1 \mathrm{~Hz}$ to $10 \mathrm{MHz}$. The samples in the form of discs with a diameter of $13 \mathrm{~mm}$ and thickness of $2 \mathrm{~mm}$ were placed between two parallel plated electrodes. In the measurements, the complex impedance $Z^{\star}(\omega)=Z(\omega)-i Z^{\prime \prime}(\omega)$ was measured and recorded at each frequency.

The magnitude $\left|Z^{\star}\right|$ and the phase $\varphi$ of the impedance are therefore given by the following expressions: $\left|Z^{\star}\right|=$ $\sqrt{Z^{\prime}(\omega)^{2}+Z^{\prime \prime}(\omega)^{2}}$ and $\varphi=\arctan \left(\frac{Z^{\prime \prime}(\omega)}{Z^{\prime}(\omega)}\right)$; here $\omega$ is the angular frequency ( $\omega=2 \pi f, f$ is the frequency).

\section{Neutron Scattering Measurements}

The neutron scattering measurements have been performed at the neutron research facility of the Budapest Neutron Centre.
SANS has prooved to be a particularly useful characterization technique for dispersion of nanostructures, providing information on the distribution patterns of the nanoscale filler materials, representative for the whole volume of the sample. The composite samples were measured with a sample to detector distance of $1.3 \mathrm{~m}$ and $5.4 \mathrm{~m}$ and with a wavelength of $\lambda=4.88 \AA$ under ambient conditions. The scattering intensity was obtained as a function of the momentum transfer (Glatter \& Kratky, 1982):

$$
Q=\frac{4 \pi}{\lambda} \sin 2 \theta
$$

where $2 \theta$ is the scattering angle. The covered $Q$ range was $0.01-0.3 \AA^{-1}$. The scattered intensity at small angles is proportional to the square of the contrast between the scattering length densities of the polymer matrix and the carbon nanostructures.

The scattering intensities of nanostructure aggregates obey a power law behavior: $I(q) \sim q^{-p}$.

The exponent $p$ in the equation describes the structure of the scattering units and their mass or surface organisation. For mass fractals (or volume fractals), the fractal dimension is: $D=p, 1<p<3$. For surface fractals, the fractal dimension is: $D S=6-p, 3<p<4$.

For scattering objects with smooth boundaries and a continuous change in scattering density, the exponent $p$ can also take values higher than 4 ; whereas, for smooth surfaces of sharp transition $p=4$. If $p$ is a non-integer, it is called fractal dimension, which is indicative of a disordered network of scatterers.

Based on the experience of SANS measurements of CNT dispersions in solutions and polymers, it is known that depending on their concentration, CNT usually form loose disordered three dimensional networks (Boukheir et al., 2016; Hattenhauer et al., 2015).

\section{RESULTS AND DISCUSSION}

Fig. 1 and 2, respectively, show the frequency dependence of the magnitude and the phase of the impedance of CNT/epoxy composites for different concentrations of CNT.

As shown in these figures, at low concentration of CNT $(\phi \leq 2.5 \%)$, the magnitude $\left|Z^{\star}(\omega)\right|$ of the impedance is a straight line with a negative slope and its phase $\varphi(\omega)$ is nearly constant (near to $-90^{\circ}$ ). At high concentration $(3.0 \%$ and $5.0 \%$ ), the curves for the magnitudes of the impedance may be divided into two distinct zones (Fig. 1): at low frequencies, the impedance is independent of the frequency, which suggests that the ohmic resistance plays an important role, while the capacitive effects can be neglected (Vivo et al., 2015). With increasing the frequency further, the magnitudes of the impedance decrease, which implies the impedance to 


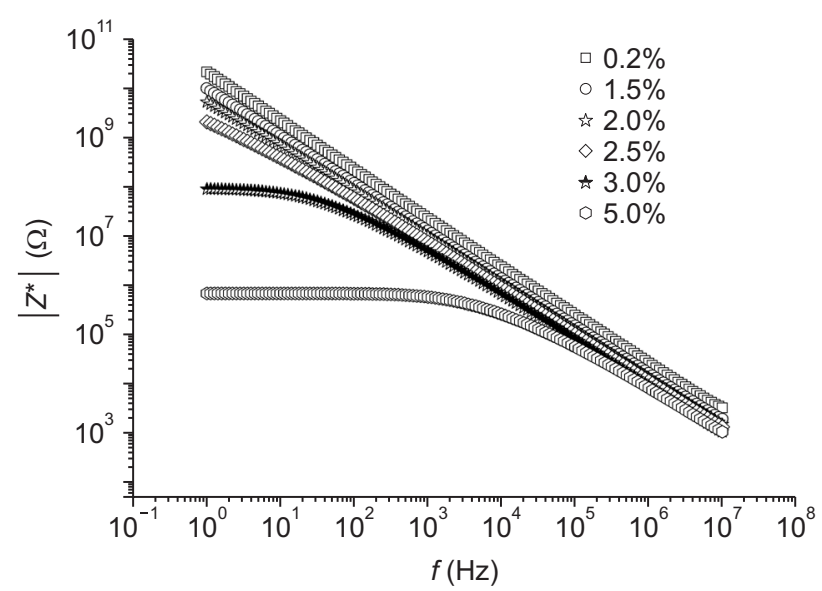

Fig. 1. Frequency dependence of the magnitude of the impedance of carbon nanotubes (CNT)/epoxy composite samples, for different concentrations of CNT.

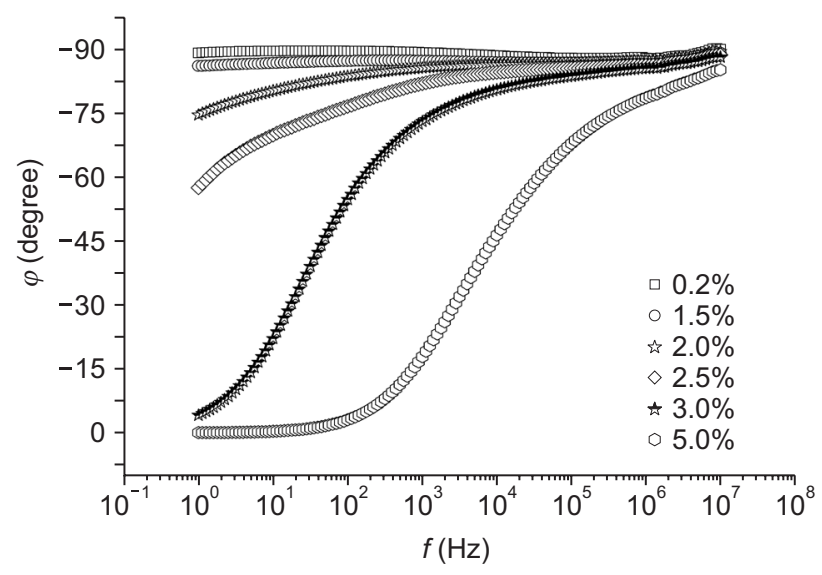

Fig. 2. Frequency dependence of the phase of the impedance of carbon nanotubes (CNT)/epoxy composite samples, for different concentrations of CNT.

take the character of a capacitor. We notice also that the phase, at high CNT concentrations, increases with frequency and approaches $-90^{\circ}$ in the high frequency region.

The plot of $Z^{\prime \prime}$ versus $Z^{\prime}$ (Nyquist diagram) for all the envisaged samples is shown in Fig. 3. From this plot a typical capacitive response is observed at low concentrations $(0.2 \%$, $1.5 \%, 2.0 \%$, and $2.5 \%$ ) showing that the dielectric properties of the polymer dominate the characteristics of the composite. At hight concentrations (3.0\%, 5.0\%), it can be observed that as CNT is added to the epoxy matrix, the enhancement of the real component of the impedance leads to the formation of a semi-circle (Bowen et al., 2014). These semicircles decrease their diameter as the CNT concentration increases, which is indicative of the lowering in the electrical resistivity of the composite due to the presence of conduction paths.

To obtain information about the dispersion and the structure
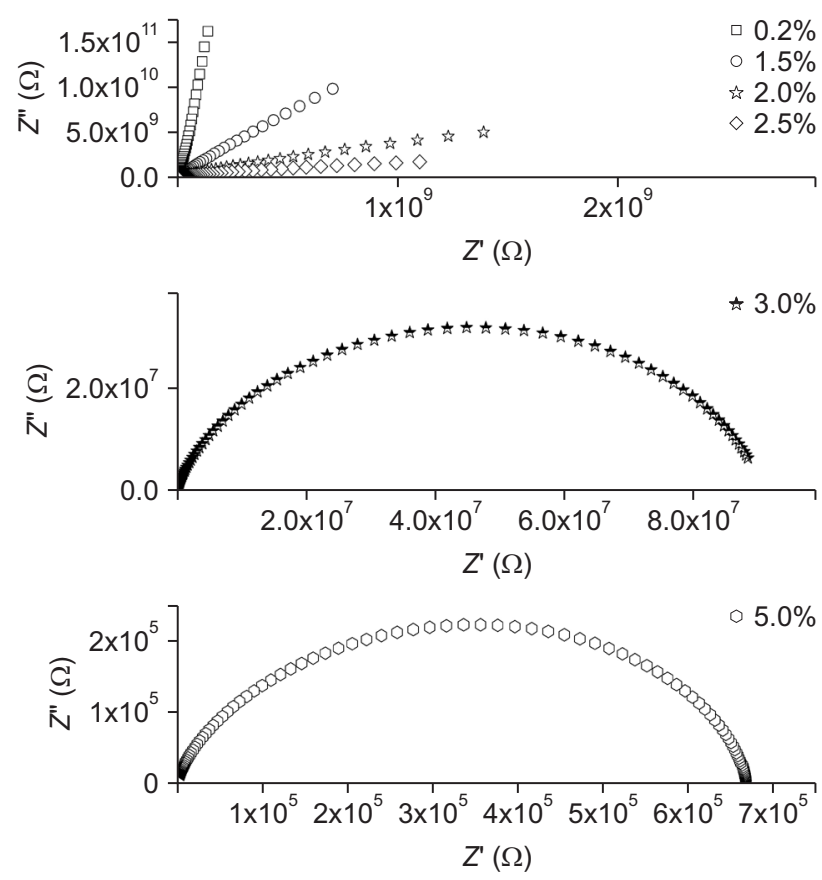

Fig. 3. Nyquist plots of carbon nanotubes (CNT)/epoxy composite samples, for different CNT concentrations.

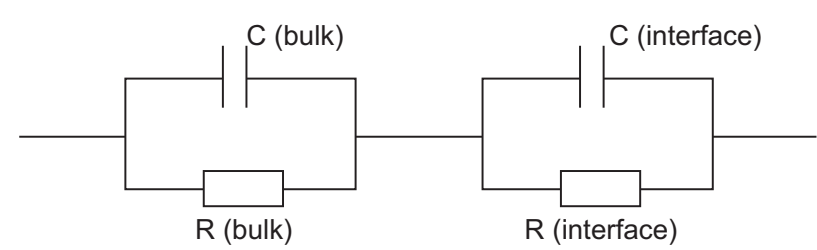

Fig. 4. Model of equivalent electric circuit of carbon nanotubes/epoxy composites.

of CNT in the epoxy matrix and to simulate the behavior of the composites, a model which contains two units in series, was used as an equivalent analogue electric circuit where, for each distinct unit, a parallel path was included comprising a resistor (R) and capacitor (C) as shown in Fig. 4; each RC element represents a Debye relaxation (Bonanos et al., 1987; Pricke, 1953; Raistrick, 1987). These two circuit units represent the contribution from the bulk composite sample and the metal-semiconductor interface. The dielectric behavior of the CNT/epoxy composites needs to be simulated and explained in a more reasonable way.

Indeed, this circuit is not sufficient to account for the interpretation of impedance spectra unless the interface capacitor in the model is substituted by the CPE, or 'fractal' capacitor. The CPE is an empirical circuit element that was introduced to account for the apparent distribution. The impedance of a CPE is given by (Rammelt \& Reinhard, 1990):

$$
\left|Z_{\text {cpe }}\right|=\sqrt{\left(Z^{\prime}\right)^{2}+\left(Z^{\prime \prime}\right)^{2}}=\frac{\omega^{-n}}{A}
$$


Table 1. Fractal dimensions of the samples measured on SANS equipment and calculated from impedence results

\begin{tabular}{lllllll}
\hline \multicolumn{1}{c}{$\phi(\%)$} & 0.2 & 1.5 & \multicolumn{1}{c}{2.0} & 2.5 & 3.0 & 5.0 \\
\hline$D$ (IS) & 2.02 & 2.04 & 2.06 & 2.11 & 2.11 & 2.21 \\
$\frac{\Delta D \text { (IS) }}{\bar{D} \text { (IS) }}(\%)$ & 3.35 & 2.39 & 1.43 & 0.96 & 0.96 & 5.74 \\
$D$ (SANS) & & & & & & \\
$\frac{\Delta D \text { (SANS) }}{\bar{D} \text { (SANS) }}(\%)$ & 0.43 & 0 & 0.43 & 0.87 & 0 & 0.43 \\
\hline
\end{tabular}

SANS, small angle neutron scattering; IS, impedance spectroscopy.

where $\omega$ is the angular frequency of the applied AC signal in the impedance experiment, $A$ is a capacitance parameter and $0<n<1$.

The power-law dependence of the CPE impedance suggests a possible relationship between CPE behavior and the fractal interface geometry of the electrode (Hernandez Creus et al., 1993; Le Mehaute \& Crepy, 1983; Nyikos \& Pajkossy, 1985; Sapoval et al., 1933).

Numerous experimental studies on various materials using different characterization techniques were performed to reveal the structure of solid aggregates by means of fractal dimension (Risović et al., 2008; Zhang et al., 2009). In some, though not all, systems studied so far, a relation (Nyikos \& Pajkossy, 1985):

$$
D=\left(\frac{1}{n}\right)+1
$$

was found to be valid between the exponent $n$ and the fractal dimension $D$. Therefore, at calculating the fractal dimension of the composites with various CNT concentrations from the IS results, equations (Eqs.) (2) and (3) were applied. Actually, Eq. (3) is consistent with the trends of a pure capacitive impedance in the smooth $(D \rightarrow 2)$ and with a constant impedance in the very rough $(D \rightarrow 3)$ limits.

Table 1 shows the values of the fractal dimensions determined by the SANS measurements and calculated from the IS method. Both employed techniques revealed that CNT/ epoxy composites exhibit surface fractal behavior (the fractal dimension of the surface is between two and three), that is, rough, colloidal and uniformly dense structures. A relative measurement uncertainty of fractal dimension: $\frac{\Delta D}{\bar{D}}$ with $\Delta D=(D-\bar{D})$ and $\bar{D}$ is average value of the fractal dimension $D$ ) was determined for both methods to substantiate the variation of $D$ by increasing the amount of CNT fillers. The almost constant values of the fractal dimension $D$ for both methods show an epoxy-CNT surface prooved to be similarly rough for all the samples, regardless the amount of CNT. The variation of the fractal dimension (determined by SANS and IS) as a function of the CNT content is depicted in Fig. 5. Concerning the values found for the fractal dimensions, it indicates that for both IS and SANS measurements, only a

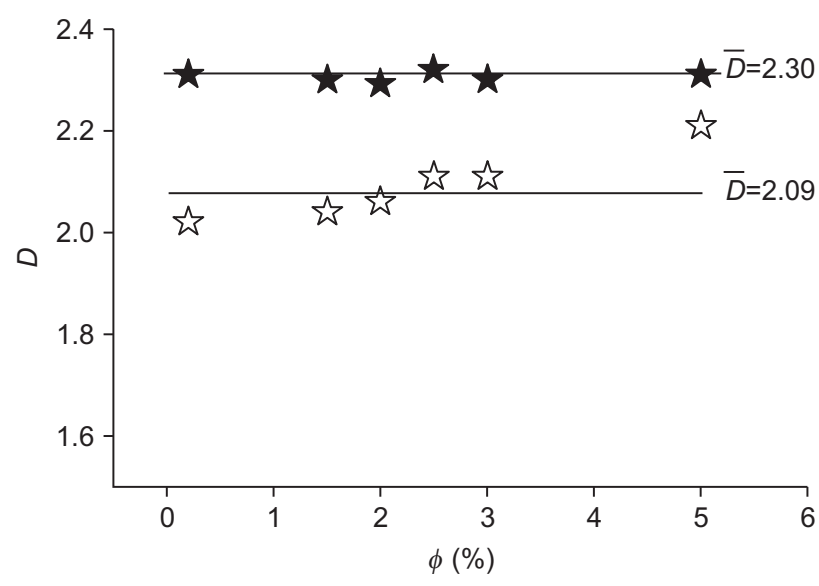

Fig. 5. Variation of the fractal dimension determined by small angle neutron scattering (SANS) and impedance spectroscopy (IS) of the composites as a function of carbon nanotubes content. Horizontal lines indicate the values of the average $(D)$ obtained by SANS and IS.

very slight difference is noticed from one CNT concentration to another. Comparing these results, we can observe that the fractal dimensions obtained by the SANS are slightly higher than those determined by the IS method. Average values of fractal dimensions $\bar{D}$, represented by an horizontal line in the Fig. 5, clearly show that also. The observed difference can be attributed to the influence of other parameters (beside the pure geometrical surface) on the CPE exponent $n$ of the IS measurements. Based on the results, it can be concluded that the IS method described in this work can be used as a simple standard tool in order to explore the fractal structure of these composites.

\section{CONCLUSIONS}

The results of this study show that the IS technique can be employed as a simple standard tool in order to analyse the fractal structure of composites. Using an equivalent circuit containing CPEs allows reproduction of the Nyquist plots of the composites. It was noticed that the IS fractal dimension increases with the increase of the CNT content, which is in good aggreement with the increase of the irregularity accompanied with the growth of the filler interface. Both employed techniques (IS and SANS) presented a fractal dimension between 2 and 3, that refer to colloidal and rough structures.

\section{CONFLICT OF INTEREST}

No potential conflict of interest relevant to this article was reported. 


\section{ACKNOWLEDGMENTS}

Financial support by the Moroccan (CNRST)-Hungarian (NKFIH) bilateral project TÉT_12_MA-1-2013-0010 and the Budapest Neutron Centre (www.bnc.hu) are gratefully acknowledged. We also thank FEDER funds through the COMPETE 2020 Program and National Funds through FCTPortuguese Foundation for Science and Technology under the project UID/CTM/50025/2013.

\section{REFERENCES}

Ali E S B and Mousa M S (2016) Switch-on phenomena and field emission from multi-walled carbon nanotubes embedded in glass. Appl. Microsc. 46, 244-252.

Allaoui A, Bai S, Cheng H M, and Bai J B (2002) Mechanical and electrical properties of a MWNT/epoxy composite. Compos. Sci. Technol. 62, 1993-1998.

Bonanos N, Steele B C H, and Butler E P (1987) Characterization of materials. In: Impedance Spectroscopy Emphasizing Solid Materials and Systems, ed. MacDonald R S, pp. 191-237, (Wiley, New York).

Boukheir S, Len A, Füzi J, Kenderesi V, Achour M E, Éber N, Costa L C, Oueriagli A, and Outzourhit A (2016) Structural characterization and electrical properties of carbon nanotubes/epoxy polymer composites. J. Appl. Polym. Sci. 134, 44514.

Bowen C R, Buschhorn S, and Adamaki V (2014) Manufacture and characterization of conductor-insulator composites based on carbon nanotubes and thermally reduced graphene oxide. Pure Appl. Chem. 86, 765.

Cooper C A, Cohen S R, Barber A H, and Wagner H D (2002) Detachment of nanotubes from a polymer matrix. Appl. Phys. Lett. 81, 3873-3875.

El-Tantawy F and Deghaidy F S (2000) Effect of iron oxide on vulcanization kinetics and electrical conductance of butyl rubber composites. Polym. Int. 49, 1371.

Glatter $O$ and Kratky $O$ (1982) Small-Angle X-ray Scattering (Academic Press, London).

Han Z and Fina A (2011) Thermal conductivity of carbon nanotubes and their polymer nanocomposites: a review. Prog. Polym. Sci. 36, 914-944.

Hattenhauer I, Tambosi P P, Duarte C A, Coelho L A F, Ramos A, and Pezzin S H (2015) Impact of electric field application during curing on epoxy-carbon nanotube nanocomposite electrical conductivity. J. Inorg. Organomet. Polym. Mater. 25, 627-634.

Hernandez Creus A, Bolzan Al, Carro P, Gonzalez S, Szdvarezza R C, and Arvia A J (1993) An ac impedance study of dendritic silver threedimensional electrodeposits. Electrochim. Acta 38, 1545.

Hopkins A R, Tomczak S J, Vij V, and Jackson A J (2011) Small Angle Neutron Scattering (SANS) characterization of electrically conducting polyaniline nanofiber/polyimide nanocomposites. Thin Solid Films 520, 1617-1620.

Kang S, Hong S, Choe C R, Park M, Rim S, and Kim J (2001) Preparation and characterization of epoxy composites filled with functionalized nanosilica particles obtained via sol-gel process. Polymer 42, 879.

Lau K, Shi S Q, and Cheng H (2002) Micro-mechanical properties and morphological observation on fracture surfaces of carbon nanotube composites pre-treated at different temperatures. Compos. Sci. Technol. 63, 1161-1164.

Le Mehaute A and Crepy G (1983) Introduction to transfer and motion in fractal media: the geometry of kinetics. Solid State Ion. 9-10, 17-30.

Lira-Olivares J, Marcano D, Lavelle C, and Sánchez F G (2000)
Determination of porosity by dielectric permitivity measurements in porous ceramics. Rev. Latinoam. Metal. y Mater. 20, 68-79.

Mandelbrot B B, Passoja D E, and Paullay A J (1984) Fractal character of fracture surfaces of metals. Nature 308, 721-722.

Nyikos I and Pajkossy T (1985) Fractal dimension and fractional power frequency-dependent impedance of blocking electrodes. Electrochim. Acta 30, 1533-1540.

Park D M, Hong W H, Kim S G, and Kim H J (2000) Heat generation of filled rubber vulcanizates and its relationship with vulcanizate network structures. Eur. Polym. J. 36, 2429.

Pricke H (1953) The Maxwell-Wagner dispersion in a suspension of ellipsoids. J. Phys. Chem. 57, 934-937.

Raistrick I D (1987) The electrical analogs of physical and chemical processes. In: Impedance Spectroscopy Emphasizing Solid Materials and Systems, ed. MacDonald R S, pp. 27-83, (Wiley, New York).

Rammelt U and Reinhard G (1990) On the applicability of a constant phase element (CPE) to the estimation of roughness of solid metal electrodes. Electrochim. Acta 35, 1045-1049.

Risović D, Poljaček S M, Furić K, and Gojo M (2008) Inferring fractal dimension of rough/porous surfaces: a comparison of SEM image analysis and electrochemical impedance spectroscopy methods. Appl. Surf. Sci. 255, 3063-3070.

Sahoo N G, Rana S, Cho J W, Li L, and Chan S H (2010) Polymer nanocomposites based on functionalized carbon nanotubes. Prog. Polym. Sci. 35, 837-867.

Salome L and Carmona F (1991) Fractal structure study of carbon-blacks used as conducting polymer fillers. Carbon N. Y. 29, 599-604.

Sapoval B, Gutfraind R, Meakin P, Keddam M, and Takenouti H (1933) Equivalent-circuit, scaling, random-walk simulation, and an experimental study of self-similar fractal electrodes and interfaces. Phys. Rev. E 4815, 3333-3344.

Tagmouti S, Bouzit S E, Costa L C, Graça M P F, and Outzourhit A (2015) Impedance spectroscopy of nanofluids based on multiwall carbon nanotubes. Spectrosc. Lett. 48, 761-766.

Tapasztó O, Lemmel H, Markó M, Balázsi M, Balázsi C, and Tapasztó $L$ (2014) The influence of sintering on the dispersion of carbon nanotubes in ceramic matrix composites. Chem. Phys. Lett. 614, $148-150$

Vivo B D, Lamberti P, Spinelli G, Tucci V, Guadagno L, and Raimondo $M$ (2015) The effect of filler aspect ratio on the electromagnetic properties of carbon-nanofibers reinforced composites. J. Appl. Phys. 118, 064302.

Zhang J, Mine M, Zhu D, and Matsuo M (2009) Electrical and dielectric behaviors and their origins in the three-dimensional polyvinyl alcohol/MWCNT composites with low percolation threshold. Carbon N. Y. 47, 1311-1320 